\title{
A comparative study of effectiveness, safety and cost effectiveness of olanzapine, risperidone and aripiprazole therapy in schizophrenia
}

\author{
Pooja R. Kanani, Ajita Pillai*
}

Department of Pharmacology, P.D.U. Government Medical College, Rajkot, Gujarat, India

\author{
Received: 13 February 2020 \\ Revised: 06 April 2020 \\ Accepted: 09 April 2020 \\ *Correspondence: \\ Dr. Ajita Pillai, \\ Email: ajitaprem@yahoo.co.in
}

Copyright: $@$ the author(s), publisher and licensee Medip Academy. This is an open-access article distributed under the terms of the Creative Commons Attribution Non-Commercial License, which permits unrestricted non-commercial use, distribution, and reproduction in any medium, provided the original work is properly cited.

\begin{abstract}
Background: Schizophrenia is the most common psychotic disorder and responsible for approximately half of longterm psychiatric hospitalizations. Antipsychotic medications reduce the psychotic symptoms and prevent relapses. The choice of drug for treatment of schizophrenia depends on many issues, including effectiveness, cost, side-effect burden, availability, and tolerability. Many studies have compared antipsychotic drugs with one another, but no broad consensus has been reached. Our study compares the clinical effectiveness, safety and cost effectiveness of atypical antipsychotics in our setting.

Methods: This was an observational, prospective study in which schizophrenia patients receiving either olanzapine, risperidone or aripiprazole were enrolled. Patients were followed up for 3 months. Evaluation of effectiveness was done by analysing mean reduction in PANSS score. Analysis of ADRs was done using WHO causality scale and Hartwig and Siegel severity scale. Cost analysis was done by comparing all three groups in term of cost range of antipsychotic drugs per improvement in PANSS score during the study period.

Results: In the present study, the average dose of antipsychotic drugs received by a patient per day was $8.83 \pm 2.98 \mathrm{mg}$ in olanzapine group, $4.76 \pm 1.12 \mathrm{mg}$ in risperidone group and $20.43 \pm 8.5 \mathrm{mg}$ in aripiprazole group. Mean reduction in PANSS score from baseline to 12 weeks was $23.79 \%$ in olanzapine group, $25.41 \%$ in risperidone group and $24.65 \%$ in aripiprazole group.

Conclusions: All the groups were equally effective in reduction in PANSS score while risperidone was the most cost effective.
\end{abstract}

Keywords: Schizophrenia, Atypical antipsychotics, PANSS, Safety, Cost effectiveness

\section{INTRODUCTION}

Schizophrenia is a severe and debilitating mental disorder that affects approximately $0.3-0.7 \%$ of the population. ${ }^{1}$ and is one of the leading causes of years lived with disability worldwide. ${ }^{2}$ People with schizophrenia present changes in thinking, feeling, and behaviour. Its manifestations are usually divided into positive symptoms (auditory hallucinations, delusions, disorganized thought, disorganized behaviour, and catatonia.), negative symptoms (alogia, flattened affect, anhedonia and avolition) and cognitive symptoms like problems with working memory and executive functioning. ${ }^{3}$ Antipsychotic medication is the main therapeutic intervention for schizophrenia. The firstgeneration 'conventional' antipsychotic drugs like trifluoperazine, haloperidol etc. are high-affinity antagonists of dopamine $\mathrm{D}_{2}$ receptors. These drugs are most effective against psychotic symptoms but have high rates of neurologic side effects, such as extrapyramidal signs and tardive dyskinesia. ${ }^{4}$ The introduction of secondgeneration, or 'atypical,' antipsychotic drugs promised enhanced efficacy and safety. ${ }^{5}$ These new medications 
differ from the conventional agents by demonstrating clinical efficacy while producing fewer extra-pyramidal symptoms (EPS); they are beneficial in treating negative symptoms and may also improve the neurocognitive deficits associated with schizophrenia. ${ }^{6}$ However, different side effects have been seen with individual agents, including weight gain and somnolence, which can decrease compliance and persistency, leading to increased relapse. Among second-generation medicines, the question of which antipsychotic should be preferred for treatment of psychotic disorders is controversial. Clear evidence-based hierarchies of the comparative efficacy, safety, risk of discontinuation and side-effects of secondgeneration antipsychotic (SGA) medications are necessary in order to recommend an antipsychotic medication in clinical practice. ${ }^{7,8}$ The primary objective of this study was to assess the effectiveness of atypical antipsychotic drugs among schizophrenics while secondary objectives were to analyse adverse drug reactions and cost effectiveness of atypical antipsychotic drugs.

\section{METHODS}

This was an observational, prospective study carried out in the department of psychiatry of P.D.U. government hospital, Rajkot, Gujarat from September, 2016 to February, 2018. Ethical approval was obtained from the Institutional Ethics Committee (date of approval27/09/2016, letter no.-19013).

Patients aged 16 to 65 years, of either gender, diagnosed with schizophreniform disorder or schizophrenia according to DSM-IV, with $\geq 4$ score of at least two characteristics positive symptoms of positive and negative syndrome scale (PANSS) score and receiving either olanzapine or risperidone or aripiprazole were included in the study.

Patients with other concomitant psychiatric illness (e.g. bipolar mood disorder or schizoaffective disorder) and receiving more than one antipsychotic drug were excluded from the study.

Data (demographic and clinical data) was extracted from the case file of the patient after obtaining informed written consent of the patient or patient's guardian and recorded in a pre-approved case record form.

Patients were followed up at 4, 8 and 12 weeks after recruitment. For assessment of effectiveness of antipsychotic therapy, PANSS was used at each visit by the attending psychiatrist. For assessment of safety, analysis of adverse drug reaction was done using WHO causality scale and Hartwig and Siegel severity scale. Cost effectiveness was calculated as the minimum and maximum cost of an antipsychotic drug required for 1point reduction in PANSS score and is represented as cost range per PANSS improvement.

\section{Statistical analysis}

All statistical analysis was performed by using MS excel2013 and past software version 3.4.1. Normality of the data was tested by Shapiro-Wilk test. Between the group's comparison of data with normal distribution was done by ANOVA whereas comparison of data with abnormal distribution was done by Kruskal-Wallis test. Effectiveness of antipsychotics was analysed by statistically significant difference in PANSS score from baseline to 12 weeks follow up period in each group using repeated measurement ANOVA. For all statistical analysis $\mathrm{p}<0.05$ was considered statistically significant. For cost analysis, all three groups were compared on the basis of money spent on antipsychotic treatment by the government in relation to improvement in PANSS score during the study period.

\section{RESULTS}

According to the inclusion criteria, a total of 95 patients were enrolled in the study, out of which 87 patients who completed at least one follow-up were analysed while 8 patients who did not complete even one follow-up were not analysed.

Mean age of patients in our study was $31.267 \pm 9.64$ years. Schizophrenia was more common in age group of 26-35 years which included $45.97 \%$ of the patients. Out of 87 patients, 54 (62\%) were males and 33 (38\%) were females with male: female ratio of 1.63:1. There was no statistically significant difference in age distribution among males and females $(\mathrm{p}=0.1248)$.

In schizophrenia patients, personal history of addiction such as tobacco chewing, smoking and alcohol drinking was analyzed. Out of 87 patients, 34 patients (39.08\%) had history of various addictions. Among the patients with history of addiction, majority of patients $(52.94 \%)$ was addicted to tobacco chewing.

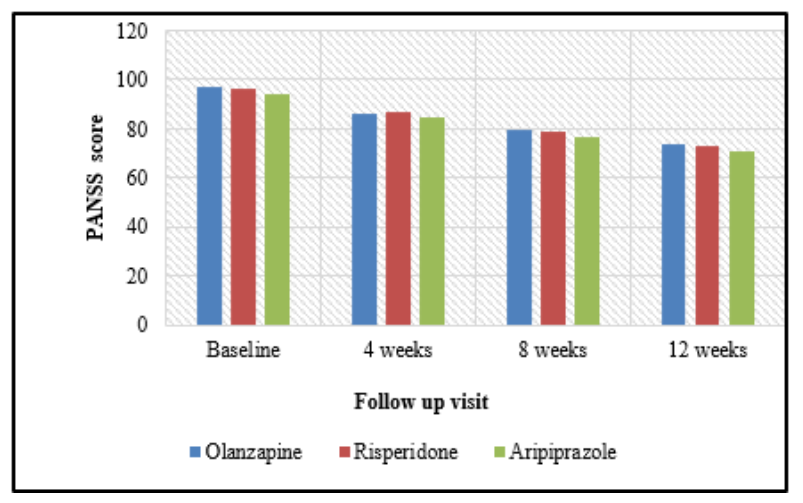

Figure 1: Comparison of PANSS score.

For analysis of effectiveness, patients were divided into 3 groups: Olanzapine $(n=31)$, Risperidone $(n=31)$ and Aripiprazole $(n=25)$. Average dose of antipsychotic drugs received by a patient per day was $8.83 \pm 2.98 \mathrm{mg}$ in 
olanzapine group, $4.76 \pm 1.12 \mathrm{mg}$ in risperidone group and $20.43 \pm 8.5 \mathrm{mg}$ in aripiprazole group. It was found that benzodiazepines were concomitantly prescribed in 24 patients in olanzapine group, 20 patients in risperidone group and 19 patients in aripiprazole group.

Assessment of effectiveness of individual antipsychotic drug was done by comparing improvement in PANSS score from baseline to 12 weeks in each group which was statistically significant in all three groups. When PANSS score of all 3 group at each follow visit was compared, between the group comparisons was found to be statistically insignificant (Table 2).

In the present study, total 22 adverse drug reactions were reported, out of which, 16 ADRs were related to antipsychotic drugs and 6 ADRs were related to benzodiazepines. Most common adverse drug reaction was extrapyramidal syndrome (hand tremors and lip smacking) (37.5\%) followed by headache and weight gain. Maximum number of adverse drug reactions $(36.36 \%)$ were seen in patients receiving olanzapine. Most common adverse drug reaction seen in patients receiving olanzapine was hand tremors. According to Hartwig and Siegel scale, all the adverse drug reactions were of mild category (level 1-3).
Table 1: Demographic characteristics $(n=87)$.

\begin{tabular}{|ll|}
\hline Demographic characteristic & N $(\%)$ \\
\hline Age (years) (Mean \pm SD) & $31.26 \pm 9.64$ \\
\hline Male: female ratio & $1.63: 1$ \\
\hline Education level & \\
\hline Illiterate & $16(18.39)$ \\
\hline Primary level & $23(26.43)$ \\
\hline Secondary level & $12(13.79)$ \\
\hline Higher secondary & $21(24.13)$ \\
\hline Graduates & $15(17.24)$ \\
\hline Area of domicile & \\
\hline Rural & $28(32.18)$ \\
\hline Suburban & $22(23.45)$ \\
\hline Urban & $37(42.52)$ \\
\hline History of addiction $(\mathbf{n}=\mathbf{3 4 )}$ & \\
\hline Tobacco chewing & $18(52.94)$ \\
\hline Alcohol & $4(11.76)$ \\
\hline Smoking & $7(20.58)$ \\
\hline Tobacco and smoking & $3(8.82)$ \\
\hline Tobacco and alcohol & $2(5.88)$ \\
\hline
\end{tabular}

Table 2: Assessment of effectiveness.

\begin{tabular}{|lllll|}
\hline Variables & Olanzapine & Risperidone & Aripiprazole & P value \\
\hline Number of participants & 30 & 29 & 22 & - \\
\hline Age in years (mean $\mathbf{4}$ SD) & $32.7 \pm 8.84$ & $34.62 \pm 12.96$ & $30.86 \pm 8.59$ & 0.457 \\
\hline Duration of illness in months(median) & 12 & 7.5 & 9 & 0.5138 \\
\hline PANSS score & & & & \\
\hline Baseline & $97.2 \pm 10.23$ & $94.37 \pm 8.29$ & $95.95 \pm 8.07$ & 0.4878 \\
\hline 4 weeks & $85.93 \pm 8.05$ & $84.38 \pm 6.34$ & $86.13 \pm 6.13$ & 0.594 \\
\hline 8 weeks & $77.26 \pm 5.55$ & $76.9 \pm 5.94$ & $77.45 \pm 6.54$ & 0.1514 \\
\hline 12 weeks & $70.77 \pm 5.81$ & $70.13 \pm 6.13$ & $71.09 \pm 5.57$ & 0.1494 \\
\hline P value & $<0.001 *$ & $<0.001 *$ & $<0.001 *$ & - \\
\hline $\begin{array}{l}\text { Reduction in PANSS score from baseline } \\
\text { to end of the study }(\%)\end{array}$ & $23.79 \%$ & $25.41 \%$ & $24.65 \%$ & - \\
\hline
\end{tabular}

Table 3: Assessment of cost effectiveness of the therapy.

\begin{tabular}{|llll|}
\hline Variables & Olanzapine & Risperidone & Aripiprazole \\
\hline Total dose required & 204 tablets & 108 tablets & 107 tablets \\
\hline Cost (Rs/10 tablets) & 1.89 & 2.25 & 8.3 \\
\hline Total cost of treatment (120 days) & Rs. 52.85 & Rs. 43.55 & Rs. 142.35 \\
\hline Cost of antipsychotic drugs & & & \\
\hline Cost of antipsychotic drugs (120 days) & Rs. 38.56 & Rs. 24.3 & Rs. 88.81 \\
\hline Minimum improvement* in PANSS score & 7 & 9 & 14 \\
\hline Maximum improvement* in PANSS score & 38 & 40 & 35 \\
\hline Maximum cost per PANSS improvement* & Rs. 5.5 & Rs. 2.7 & Rs. 6.34 \\
\hline Minimum cost per PANSS improvement* & Rs. 1.14 & Rs. 0.6 & Rs. 2.5 \\
\hline Cost range per PANSS improvement* & Rs. 1.14-5.5 & Rs. 0.6-2.7 & Rs. 2.5 - 6.34 \\
\hline
\end{tabular}


In our study, cost of the antipsychotic treatment borne by government was calculated. Cost analysis revealed that total cost of 120 days of therapy was highest with aripiprazole followed by olanzapine. Cost per PANSS score improvement was lowest with risperidone group. However, there was no statistically significant difference between cost of olanzapine and risperidone (Table 3 ).

\section{DISCUSSION}

Choice among the antipsychotic drugs is based mainly on the differences in adverse effects and possible differences in efficacy.

In the present study, the gender distribution and mean age of patients is comparable to that reported in earlier studies. ${ }^{9-11}$ Demographic characteristics showed that the mean and standard deviation of age of the study patients was $(31.267+9.64)$ years. In an Indian epidemiological study done by Ashturkar et al, in Aurangabad, mean age for the cases was 30.26 years which is comparable to our study. ${ }^{9}$ The onset of schizophrenia usually occurs between late teens and the mid-30s. ${ }^{1}$ In our study, majority of patients $(45.97 \%)$ were in the age group of (26-35) years and a higher number of male patients $(62 \%)$ were observed with male to female ratio of 1:0.61.Similar results were found in a study done by Ashturkar et al, in which it was reported that $66.66 \%$ patients were males and $33.33 \%$ were females. ${ }^{9}$ McGrath et al studied the incidence and prevalence of schizophrenia and found that the incident was significantly higher in males compared to females; the male/female ratio median was 1:0.4. ${ }^{10}$ The onset of schizophrenia is later in women than in men, and the clinical manifestations are less severe. This may be because of the antidopaminergic influence of oestrogen. 11

In the present study, 39.08\% of the study patients had history of substance abuse. Various substances of abuse reported in our study were tobacco chewing in majority of patients $(52.94 \%)$ followed by alcohol drinking and cigarette smoking. In a study done by Kothari et al. 55\% patients had a history of substance abuse. ${ }^{12}$ The reasons for high frequency of both smoking prevalence and heavy smoking in schizophrenic patients is thought to be at least partially related to enhancement of brain dopaminergic activity, which, in turn, results in behavioural reinforcement due to stimulant effects. Cigarette smoke also increases the activity of CYP 1A2 enzymes, thus decreasing the concentration of many drugs, including clozapine and olanzapine. Schizophrenic patients who smoke may require higher dosages of antipsychotics than non-smokers. ${ }^{14}$

Antipsychotic drugs are the mainstay of the treatment of schizophrenia. The selection of an antipsychotic drug to treat patients with schizophrenia is a complex decision for which the physician must weigh individual patient's factors and numerous drug factors, including efficacy, safety, tolerability, and cost effectiveness. ${ }^{15}$ Zhang et al did meta-analysis on efficacy and safety of individual second generation versus first generation antipsychotics and found that pooled second generation antipsychotics (SGAs) were either similarly effective or modestly better than first generation antipsychotics (FGAs) regarding several efficacy and tolerability outcomes. ${ }^{16}$ Because of clear advantages in terms of fewer side effects, particularly extrapyramidal side effects, and better efficacy as proven by various clinical trials, second generation antipsychotics are preferred over first generation antipsychotics now a days.

In the present study, mean per day dosage of antipsychotic drug received by patients was $(8.87+3.34)$ $\mathrm{mg}$ in olanzapine group, $(4.77+1.12) \mathrm{mg}$ in risperidone group and $(20.98+8.91) \mathrm{mg}$ in aripiprazole group. Doses of antipsychotic drugs were similar to those used in current clinical practice. The mean baseline PANSS score was 96.96 in olanzapine group, 94.4 in risperidone group and 95.28 in aripiprazole group. There was no statistically significant difference in mean baseline PANSS score among the three groups. The mean baseline PANSS score in our study was higher than previous studies. ${ }^{17,18}$ At the end of 12 weeks, total reduction in PANSS score from baseline was $25.93 \%$ in olanzapine group, $24,89 \%$ in risperidone group and $23 \%$ in aripiprazole group. Within the group comparison revealed that reduction in total PANSS score from baseline to 12 weeks in each group was statistically significant in all three groups. In the present study, when the mean reduction in PANSS score from baseline to 12 weeks was compared among olanzapine, risperidone and aripiprazole groups, no statistically significant difference between the groups was found. This implies that all three drugs are equally efficacious.

In a meta-analysis comparing efficacy of risperidone versus other atypical antipsychotics by comparing various clinical outcome, Komossa et al, found similar results. In terms of reduction in PANSS score, head to head comparison of risperidone and aripiprazole showed both drugs to be equally efficacious, whereas comparison of risperidone and olanzapine showed olanzapine to be more efficacious. ${ }^{19}$ Conley et al compared the effectiveness of risperidone and olanzapine in 377 patients and found both the drugs equally efficacious. ${ }^{17}$ Kumar et al also reported similar results. Jindal et al compared efficacy of aripiprazole and olanzapine in a randomized double-blind controlled study. This trial demonstrated that aripiprazole was equally efficacious as olanzapine in the treatment of schizophrenia. ${ }^{20}$

Schizophrenia is associated with significant economic burden. The mean monthly costs per patient on treatment with schizophrenia is typically more than four times higher than those for the population as a whole with similar demographics but without schizophrenia. ${ }^{21}$ Among all the three drugs, aripiprazole was found to have the highest cost range per PANSS improvement 
followed by olanzapine. Cost range per PANSS improvement for risperidone was lowest (Rs. 0.6-2.7). Barbosa et al. studied the annual cost of treatment of schizophrenia in adult patients receiving atypical antipsychotics in Brazil and found that cost of olanzapine was high compared to risperidone. ${ }^{22}$

\section{CONCLUSION}

From our study, it can be concluded that all three drugs i.e. olanzapine, risperidone and aripiprazole are equally efficacious in terms of alleviating symptoms of schizophrenia. All the adverse drug reactions observed during the study period were of mild category. Majority of adverse reactions were seen in patients receiving olanzapine. Cost analysis revealed that risperidone is the most cost-effective drug.

\section{ACKNOWLEDGEMENTS}

I would like to acknowledge the faculties of psychiatry department of our institute for their cooperation throughout my study period.

Funding: No funding sources

Conflict of interest: None declared

Ethical approval: The study was approved by the Institutional Ethics Committee

\section{REFERENCES}

1. Schizophrenia spectrum and other psychotic disorders. In: Diagnostic and Statistical manual of Mental Disorder. American Psychiatric Association. 5th ed. New Delhi: CBS; 2017: 155-188.

2. Whiteford HA, Degenhardt L, Rehm J. Global burden of disease attributable to mental and substance use disorders: findings from the Global Burden of Disease Study 2010. Lancet. 2013;382:1575-86.

3. National Institute for Health and Care Excellence. Clinical guideline [CG178]. Psychosis and schizophrenia in adults: prevention and management. National Institute for Health and Care excellence; 2014.

4. Lieberman JA, Stroup TS, Evoy MJP, Swartz MS, Rosenheck RA, Perkins DO, et al. Effectiveness of Antipsychotic Drugs in Patients with Chronic Schizophrenia. Nengl J Med. 2005;353(12):1211-23.

5. Kane J, Honigfeld G, Singer J, Meltzer H. Clozapine for the treatment-resistant schizophrenic: a doubleblind comparison with chlorpromazine. Arch Gen Psychiatry. 1988;45:789-96.

6. Chavda RK, Laxmi L, Nair BS, Gandewar K. Efficacy and Tolerability of Aripiprazole in Patients with Schizophrenia and Schizoaffective Disorders. Indian J Psychiatry. 2004;46(2):150-5.

7. World Health Organization. World Report on Disability 2011. 2015. Available at: http://apps.who. int/iris/handle/10665/445758. Accessed on 26 November 2018.

8. World Health Organization. Second-generation antipsychotic medications for psychotic disorders (including schizophrenia). 2015 Available at: http://www.who.int/mental_health/mhgap/evidence/r esource/psychosis_q14.pdf?ua=1. Accessed on 26 November 2018.

9. Ashturkar MD, Dixit JV. Selected epidemiological aspects of schizophrenia: a cross sectional study at tertiary care hospital in Maharashtra. Natl J Community. 2013;4(1):65-7.

10. Grath MJ, Saha S, Welham J, Saadi O, Culey CM, Chant D. A Systematic Review of the Incidence of Schizophrenia: The Distribution of Rates and Influence of Sex, Urbanicity, Migrant Status and Methodology. Biomed Central Med. 2004;13(2):1741-7015.

11. Frankenburg FR. Schizophrenia. 2018. Available at: https://emedicine.medscape.com/article/288259overview\#a5. Accessed on 26 November 2018.

12. Kothari S, Patel P, Changulani M, Agarwal A. Efficacy, tolerability and cost effectiveness of zotepine versus olanzapine in patients of acute schizophrenia Int $\mathrm{J}$ Basic Clin Pharmacol. 2013;2(5):577-82.

13. Jim VO, Allardyce J. Schizophrenia and other psychotic disorders: The Clinical Epidemiology of Schizophrenia. In: Sadock BJ, Sadock VA, Ruiz P, editors. Kaplan and Sadock's comprehensive textbook of Psychiatry. 9th ed. New York: Wolter Kluwer| Lippincott William and willkins; 2009: 1475-1486.

14. Sagud M, Peles MA, Seler MD, Pivac N, VuksanCusa B, Brataljenovic, et al. Smoking and schizophrenia. Psychiatr Danub. 2009;21(3):371-5.

15. Franz M, Ranger J, Hanewald B, Gallhofer B, Lay B. Influences on therapist's decisions for neuroleptic treatment in schizophrenia: the role of characteristics of the patient and the physician. Pharmacopsychiatry. 2012;45(7):261-8.

16. Zhang JP, Gallego JA, Robinson DG, Malhotra AK, Kane JM, Correll CU. Efficacy and Safety of Individual Second-Generation vs First-Generation Antipsychotics in First Episode Psychosis: A Systematic Review and Meta-analysis. Int $\mathbf{J}$ Neuropsychopharmacol. 2013;16(6):1205-18.

17. Conley RR, Mahmoud R. A Randomized DoubleBlind Study of Risperidone and Olanzapine in the Treatment of Schizophrenia or Schizoaffective Disorder. Am J Psychiatry. 2001;158:765-74.

18. Kumar PS, Anish PK, Rajamohan V. Olanzapine has better efficacy compared to risperidone for treatment of negative symptoms in schizophrenia. Indian $\mathbf{J}$ Psychiatry. 2016;58(3):311-6.

19. Komossa K, Kluge RC, Schwarz S, Schmid F, Hunger H, Kissling K, et al. Risperidone versus other atypical antipsychotics for Schizophrenia. Cochrane Database Syst Rev. 2014;(1):CD006626. 
20. Jindal KC, Singh JP, Munjal V. Aripiprazole versus olanzapine in the treatment of schizophrenia: A clinical study India. Int $\mathbf{J}$ Psychiatry Clin Pract. 2013;17(2):21

21. Fitch K, Iwasaki K, Villa KF. Resource utilization and cost in a commercially insured population with schizophrenia. Am Health Drug Benefits. 2014;7(1):18-26.

22. Barbosa WB, Costa JO, Lemos LL, Gomes RM, Oliveira HN, Ruas CM, et al. Costs in the Treatment of Schizophrenia in Adults Receiving Atypical Antipsychotics: An 11 Years Cohort in Brazil. Appl Health Econ Health Policy. 2018;16(5):697-709.

Cite this article as: Kanani PR, Pillai A. A comparative study of effectiveness, safety and cost effectiveness of olanzapine, risperidone and aripiprazole therapy in schizophrenia. Int $\mathbf{J}$ Basic Clin Pharmacol 2020;9:786-91. 